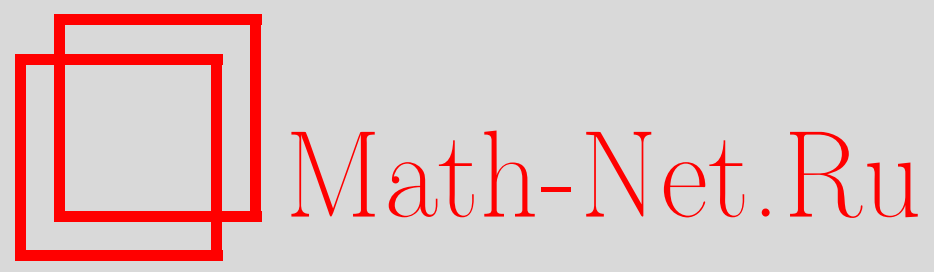

А. А. Булатов, Абстрактные свойства класса интервалов решеток замкнутых классов, Дискрет. матем., 2000, том 12, выпуск 3, 95-113

DOI: https://doi.org/10.4213/dm337

Использование Общероссийского математического портала Math-Net.Ru подразумевает, что вы прочитали и согласны с пользовательским соглашением http://www.mathnet.ru/rus/agreement

Параметры загрузки:

IP : 3.85 .7 .115

26 апреля 2023 г., 18:13:52

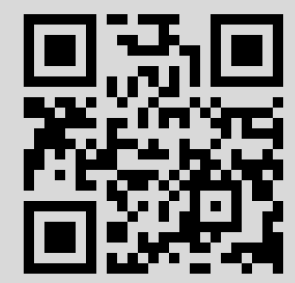




\title{
Абстрактные свойства класса интервалов решеток замкнутых классов
}

\author{
(C) 2000 г. А. А. Булатов
}

\begin{abstract}
Рассматривается решетка $\mathscr{L}_{k}$ замкнутых классов, содержащих все проекции (клонов), на $k$-элементном множестве. Доказано, что для любого $k \geqslant 2$ счетная прямая степень решетки $\mathscr{L}_{k}$ изоморфна интервалу решетки $\mathscr{L}_{k+3}$. Отсюда вытекает, в частности, что класс всех подрешеток (интервалов) решеток клонов замкнут относительно взятия счетных прямых степеней.
\end{abstract}

\section{1. Введение}

Статья посвящена изучению свойств класса подрешеток и класса решеток, изоморфных интервалам решеток замкнутых классов функций на конечном множестве. Как известно, структура решетки замкнутых классов, за исключением решетки замкнутых классов булевых функций, весьма сложна и не оставляет надежд на получение сколь-нибудь приемлемого описания. Мы надеемся, что, как и при изучении других решеток, исследование класса подрешеток и класса интервалов прояснит и строение решетки замкнутых классов в целом.

К настоящему времени эти два класса практически не исследованы. Исключением является решетка замкнутых классов булевых функций, для которой имеется исчерпывающее описание [1]. Кроме того, известен обширный класс решеток, содержащий, в частности, все конечные решетки, вложимых в решетку замкнутых классов функций, на множестве более, чем с тремя элементами [2]. Данная работа продолжает изучение класса решеток, вложимых в решетки замкнутых классов, начатое в $[2,3,4]$.

\section{2. Предварительные замечания и формулировка результатов}

В статье используются известные понятия, их можно найти в [6, 7]. Напомним лишь некоторые обозначения. Для конечного множества $A,|A|=k$, через $O_{A}$ обозначается множество всех функций на $A$. Число переменных функции $f$ обозначается через $\operatorname{ar} f$. Решетка всех клонов обозначается через $\mathscr{L}_{A}$. Она является подрешеткой, а точнее, главным фильтром решетки замкнутых классов, порожденным классом всех проекций. Это обстоятельство позволяет распространить все результаты, упомянутые в статье, на решетки замкнутых классов. Поскольку решетки клонов 
на равномощных множествах изоморфны, мы будем использовать обозначение $\mathscr{L}_{k}$ вместо $\mathscr{L}_{A}$. Более подробные сведения о решетках клонов можно почерпнуть в [6, 7], а здесь мы изложим имеющиеся сведения лишь о классе подрешеток решетки клонов. Понятия и факты теории решеток читатель может найти в книге [5].

Решетки клонов $\mathscr{L}_{k}$ при $k \geqslant 3$ континуальны (см. [8]), и имеется целый ряд результатов, некоторые из них будут упомянуты ниже, свидетельствующих о том, что структура решеток клонов в этом случае весьма сложна. Изучение класса подрешеток (интервалов) решетки клонов представляется поэтому весьма интересной и привлекательной задачей. Существует две задачи, на решение которых направлена данная работа. Эти задачи соответствуют двум направлениям, разрабатываемым в исследовании указанных классов.

Во-первых, вопросы, связанные с вложением решеток $\mathscr{L}_{k}$ друг в друга. В настоящее время известно лишь два несложно проверяемых, но красивых факта, относящихся к этой проблеме (см. [7], п. 3.3, с. 86). А именно, для любого натурального $k$ решетка $\mathscr{L}_{k}$ изоморфна интервалу в $\mathscr{L}_{k+1}$, причем верхняя граница интервала является коатомом в $\mathscr{L}_{k+1}$. Далее, прямое произведение решеток $\mathscr{L}_{k}$ и $\mathscr{L}_{l}$ изоморфно интервалу в $\mathscr{L}_{k l}$, причем нижняя граница интервала есть атом в $\mathscr{L}_{k l}$, а верхняя пересечение двух коатомов. Отсюда следует, что класс подрешеток (как и класс интервалов) решеток клонов замкнут относительно конечных прямых произведений. Отметим также, что не имеется никакой информации относительно вложения $\mathscr{L}_{k}$ в $\mathscr{L}_{m}$ при $m<k$. Неизвестно, в частности, решение следующей проблемы: существует ли число $k$ такое, что для любого $m$ решетка $\mathscr{L}_{m}$ изоморфно вложима в $\mathscr{L}_{k}$ ?

Во-вторых, расширение запаса решеток и классов решеток, вложимых или представимых интервалами решеток клонов. Бо́льшая часть результатов в этом направлении, кроме изолированных примеров конкретных подрешеток, связана с декартовым произведением решеток. Хронологически первым результатом такого рода следует, по-видимому, считать статью [9]. В ней показано, что решетка подмножеств счетного множества (т.е. счетная декартова степень двухэлементной решетки) изоморфна некоторому интервалу в $\mathscr{L}_{k}, k \geqslant 3$. Усилением этого факта является утверждение из [10], гласящее, что счетная декартова степень цепи типа $\omega+1$ изоморфна некоторому интервалу в $\mathscr{L}_{k}, k \geqslant 3$. Далее, в [4] для произвольной последовательности конечных полурешеток указан интервал в $\mathscr{L}_{3}$, изоморфный декартову произведению их решеток подполурешеток. Отсюда следует, что такой интервал существует и в любой $\mathscr{L}_{k}$ при $k \geqslant 3$. Наконец, как показано в [2], декартово произведение последовательности произвольных конечных решеток изоморфно вложимо в $\mathscr{L}_{k}$ для $k \geqslant 4$.

Таким образом, во всех случаях возникают классы решеток, замкнутые относительно счетных декартовых произведений. Как показывает следующая теорема, являющаяся основным результатом статьи, это обстоятельство не случайно. Кроме того, теорема дает новую информацию о вложении решеток $\mathscr{L}_{k}$ друг в друга, усиливая утверждение о вложимости прямых произведений.

Теорема 1. Для любого $k \geqslant 2$ счетная степень решетки $\mathscr{L}_{k}$ изоморфна некоторому интервалу в решетке $\mathscr{L}_{k+3}$.

Применительно к классу подрешеток решеток клонов теорема влечет следующее утверждение.

Следствие 1. Класс подрешеток (интервалов) решеток клонов замкнут относи- 
тельно счетных прямих степеней.

К сожалению, из теоремы не следует замкнутость класса подрешеток (интервалов) решеток клонов относительно счетных прямых произведений. Таким образом, остается открытой следующая проблема: замкнут ли класс подрешеток (интервалов) решеток клонов относителъно счетных прлмых произведений?

Отметим еще, что, как нетрудно видеть, две сформулированные проблемы эквивалентны.

\section{3. Метод доказательства}

Для доказательства теоремы мы используем соответствие Галуа между клонами функций и отношений (см. [7], п.1.2, с.53). Множество всех $n$-местных отношений на множестве $A$ обозначим через $R_{A}^{(n)}$ (для отношения $\rho \in R_{A}^{(n)}$ мы будем также писать $\operatorname{ar} \rho=n$ ). Пусть, кроме того,

$$
R_{A}=\bigcup_{n \in \mathbb{N}} R_{A}^{(n)}
$$

Элемент отношения будем называть кортежем и использовать для его обозначения полужирный шрифт, например, $\mathbf{a}=\left(a_{1}, \ldots, a_{n}\right)=\left(a_{i}\right)_{i \in N_{n}}$, где $N_{n}=\{1, \ldots, n\}$. Нам придется рассматривать отношения, чьи координаты индексированы элементами некоторого множества $I \subseteq \mathbf{N}$. Кортежи такого отношения будем обозначать $\left(a_{i}\right)_{i \in I}$. При этом множество $I$ (состоящее из натуральных чисел) предполагается естественным образом упорядоченным.

Как обычно, для $C \subseteq O_{A}, T \subseteq R_{A}$ через Inv $C$ обозначается множество всех отношений, сохраняемых функциями из $C$, а через $\operatorname{Pol} T-$ множество всех функций, сохраняющих все отношения из $T$. Множествами вида $\operatorname{Pol} T$ являются в точности клоны (см. [7], п. 1.2, с. 53). Множества отношений вида $\operatorname{Inv} C$ также можно охарактеризовать на языке замкнутости относительно некоторых операций. Мы приведем небольшой список таких операций. Часть этих операций используется в [11]. Нам будут удобны несколько отличные от использованных в [11] обозначения, которые понадобятся нам ниже. Пусть $\operatorname{ar} \rho=n, \operatorname{ar} \sigma=l$.

Проекция. Пусть $I \subseteq N_{n}-$ множество координат. Для кортежа $\mathbf{a}=\left(a_{1}, \ldots, a_{n}\right)$ через $\left.\mathbf{a}\right|_{I}$ обозначим кортеж $\left(a_{i}\right)_{i \in I}$. Проекцией $\rho$ на $I$ называется отношение

$$
\left.\rho\right|_{I}=\left\{\left.\mathbf{a}\right|_{I} \mid \mathbf{a} \in \rho\right\}
$$

Прямое произведение. Прямым произведением отношений $\rho$ и $\sigma$ называется отношение

$$
\rho \times \sigma=\left\{\left(a_{1}, \ldots, a_{n}, b_{1}, \ldots, b_{l}\right) \mid\left(a_{1}, \ldots, a_{n}\right) \in \rho,\left(b_{1}, \ldots, b_{l}\right) \in \sigma\right\} .
$$

Перестановка координат. Для перестановки $\pi$ множества $N_{n}$ отношение

$$
\pi(\rho)=\left\{\left(a_{\pi(1)}, \ldots, a_{\pi(n)}\right) \mid\left(a_{1}, \ldots, a_{n}\right) \in \rho\right\}
$$

называется отношением, полученным из $\rho$ в результате перестановки координат $\pi$. 
Отождествление координат. Отождествлением координат $i$ и $j, i<j$, отношения $\rho$ называется операция, определенная следующим образом:

$$
\nabla_{i, j}(\rho)=\left\{\left(a_{1}, \ldots, a_{n-1}\right) \mid\left(a_{1}, \ldots, a_{i}, \ldots, a_{j-1}, a_{i}, a_{j}, \ldots, a_{n-1}\right) \in \rho\right\} .
$$

Удвоение координаты. Удвоением $i$-й координаты отношения $\rho$ называется операция, определенная следующим образом:

$$
D_{i}(\rho)=\left\{\left(a_{1}, \ldots, a_{i-1}, a_{i}, a_{i}, a_{i+1}, \ldots, a_{n}\right) \mid\left(a_{1}, \ldots, a_{n} \in \rho\right\} .\right.
$$

Далее тривиальным называется отношение вида $v_{n}^{\sigma}$, где $\sigma$ - отношение эквивалентности на множестве $N_{n}$, причем кортеж $\left(a_{1}, \ldots, a_{n}\right)$ содержится в $v_{n}^{\sigma}$ тогда и только тогда, когда $a_{i}=a_{j}$ для всех пар $(i, j) \in \sigma$. Частными случаями тривиальных отношений служат универсальное отношение $v_{n}(\sigma-$ отношение равенства) и обобщенное отношение равенства $\omega_{n}$ ( $\sigma$ - универсальное отношение).

Известно, что каждое множество вида Inv $C$ замкнуто относительно всех из перечисленных операций и содержит все тривиальные отношения. В [11] было доказано и обратное, а именно, каждое множество отношений, содержащее отношение равенства и замкнутое относительно отождествления координат, проекций, прямых произведений и перестановок координат имеет вид Inv $C$. Такие замкнутые множества отношений называются также клонами отношений.

Все клоны отношений на $k$-элементном множестве $A$ образуют полную решетку $\mathscr{L}_{k}^{\partial}$, которая дуально изоморфна решетке клонов $\mathscr{L}_{k}$. Этим обстоятельством мы и воспользуемся для доказательства нашей теоремы.

Замкнутость множества отношений можно выразить также на языке математической логики ([7], п. 2.1, с.64). Каждому $n$-местному отношению $\rho$ естественным образом сопоставим $n$-местный предикат $P_{\rho}$. Каждой из перечисленных выше операций соответствует $\exists \wedge$-формула исчисления предикатов. Например, справедливо равенство

$$
P_{\rho^{\prime}}\left(x_{1}, \ldots, x_{n-1}\right)=\exists x_{n} P_{\rho}\left(x_{1}, \ldots, x_{n}\right),
$$

где $\rho^{\prime}=\left.\rho\right|_{N_{n-1}}$. Более того, для любых отношений $\rho_{\alpha}, \alpha \in \Lambda$, где $\Lambda$ - конечное множество, $\operatorname{ar} \rho_{\alpha}=n_{\alpha}$, произвольных $n, m \in \mathbf{N}$ и произвольных отображений $\pi: N_{n} \rightarrow N_{m}$ и $\pi_{\alpha}: N_{n_{\alpha}} \rightarrow N_{m}, \alpha \in \Lambda$, отношение $\rho$, определяемое формулой

$$
P_{\rho}\left(x_{\pi(1)}, \ldots, x_{\pi(n)}\right)=\exists\left(x_{i}\right)_{i \in N_{m} \backslash \pi\left(N_{n}\right)} \bigwedge_{\alpha \in \Lambda} P_{\rho_{\alpha}}\left(x_{\pi_{\alpha}(1)}, \ldots, x_{\pi_{\alpha}\left(n_{\alpha}\right)}\right)
$$

содержится в клоне отношений $\left\langle\left\{\rho_{\alpha} \mid \alpha \in \Lambda\right\}\right\rangle$. Можно проверить и обратное.

Предложение 1. Если $\rho \in\langle S\rangle, S \subseteq R_{A}$, то для некоторого конечного $\Lambda$ и отношений $\rho_{\alpha} \in S, \alpha \in \Lambda$, найдется формула вида (1), представляющая отношение $\rho$.

Кортеж $\left(a_{1}, \ldots, a_{m}\right)$, для которого истинна бескванторная часть формулы (1), то есть

$$
\bigwedge_{\alpha \in \Lambda} P_{\rho_{\alpha}}\left(x_{\pi_{\alpha}(1)}, \ldots, x_{\pi_{\alpha}\left(n_{\alpha}\right)}\right)
$$

будем называть допустимым для (1).

'На самом деле, при изучении клона отношений, порожденного множеством отношений, можно несколько сузить класс рассматриваемых $\exists \wedge$-формул. Для этого 
заметим сначала, что если отображение $\pi$ не инъективно, например, $\pi(i)=\pi(j)$, то для каждого кортежа $\mathbf{a} \in \rho$ выполнено равенство $a_{i}=a_{j}$. Однако, в этом случае $\rho$ можно получить из отношения $\left.\rho\right|_{I \backslash\{j\}}$ при помощи удвоения координат. Поэтому нам достаточно рассматривать лишь отношения, у которых никакая пара координат не обладает указанным свойством, а значит, можно полагать отображение $\pi$ инъективным. При этом предположении мы можем переопределить подходящим образом отображения $\pi, \pi_{\alpha}, \alpha \in \Lambda$, так, чтобы выполнялось условие $\pi(i)=i$ для всех $i \in N_{n}$. В дальнейшем мы всегда будем предполагать, что $\pi$ имеет такой вид.

\section{4. Индексации и кодирующие отношения}

\section{1. Некоторые соглашения}

В доказательстве нам понадобятся многие объекты различной природы. Примем некоторые соглашения относительно обозначений. Мы рассматриваем отношения и функции на множествах $E_{k}=\{0,1, \ldots, k-1\}$ и $E_{k+3}$. Отношения на множестве $E_{k}$ мы будем обозначать буквой $\rho$, возможно с индексами, а отношения на $E_{k+3}$ буквой $\tau$. Множества натуральных чисел (обычно это будут множества координат отношений) обозначаются прописными латинскими буквами, предпочтительно из середины алфавита. Наконец, нам часто будут нужны отображения множеств натуральных чисел друг в друга, такие отображения будут обозначаться буквами $\gamma, \mu$.

\section{2. Несколько специальных кортежей}

Пусть $p$ - простое число, большее 5, и $r \in \mathbf{N}$. Рассмотрим кортежи

$$
\mathbf{v}_{j}^{r}=(k+1, \ldots, k+1, k, k+1, \ldots, k+1)
$$

где $k$ стоит на $j$-м месте,

$$
\mathbf{s}_{j}^{r}=(k+1, \ldots, k+1, k, k, k+1, \ldots, k+1),
$$

где $k$ стоит на местах с номерами $j$ и $j+1$,

$$
\mathbf{t}^{r}=(k, k, k, k+1, \ldots, k+1)
$$

с $p^{r}$ компонентами, где $1 \leqslant j \leqslant p^{r}$ для $\mathbf{v}_{j}^{r}$ и $1 \leqslant j \leqslant p^{r}-1$ для $\mathbf{s}_{j}^{r}$. Кортеж $\mathbf{v}_{j}^{r}$ назовем $r$-выделяющим с номером $j$ или $j$-м $r$-выделяющим кортежем. Аналогично, кортеж $\mathbf{s}_{j}^{r}$ назовем $r$-связывающим кортежем с номером $j$ или, короче, $j$-м $r$-связывающим кортежем, a $\mathbf{t}^{r}-r$-титульным кортежем. Число $j$ мы будем называть номером, a $r$ - рангом указанных кортежей.

\section{3. Индексации}

Пусть $I, J$ - множества натуральных чисел и $\gamma$ - отображениє множества $I$ в множество $J$. Скажем, что кортеж $\mathbf{b}=\left(b_{j}\right)_{j \in J}$ является образом корт зжа $\mathbf{a}=\left(a_{i}\right)_{i \in I}$, a кортеж a прообразом кортежа $\mathbf{b}$ (в обозначения $\mathbf{b}=\gamma(\mathbf{a})$ или $\mathbf{a}=\gamma^{-1}(\mathbf{b})$ ), если $a_{i}=b_{\gamma(i)}$ для всех $i \in I$. Кортеж может иметь более одного образа, с сли отображение $\gamma$ не является отображением на или не иметь его вовсе, если $\gamma$ нє йьективно. В то же время прообраз всегда единствен. 
Пусть теперь $\rho-n$-местное отношение и $I \subseteq N_{n}$. Отображение $\gamma: I \rightarrow N_{p^{r}}$ называется индексацией ( $r$-индексацией или индексацией ранга $r$ ) множества $I$ координат отношения $\rho$, если для любого $j, 1 \leqslant j \leqslant p^{r}$, отношение $\left.\rho\right|_{I}$ содержит прообразы кортежей $\mathbf{v}_{j}^{r}, \mathbf{s}_{j}^{r}, \mathbf{t}^{r}$. Само множество $I$ мы назовем индексируемым ( $r$-индексируемым), если существует хотя бы одна его индексация ( $r$-индексация). Мы также будем называть отношение индексируемым ( $r$-индексируемым), если индексируемо множество всех его координат.

Простейшим примером $r$-индексируемого отношения может служить $p^{r}$-местное отношение, состоящее в точности из $r$-выделяющих, $r$-связывающих и $r$-титульного кортежей.

В завершение этого пункта приведем два легко проверяемых утверждения.

Лемма 1. Если отображение $\gamma: I \rightarrow N_{p}^{r}, I \subseteq N_{n}$, являетсл индексацией $n$-местного отношения $\rho$ и не является отображением на, то $\left.\rho\right|_{I}$ содержит кортеж $(k+1, \ldots, k+1)$.

Доказательство. Пусть $j \notin \gamma(I)$ и пусть кортеж а $\in \rho$ таков, что а $I$ - прообраз кортежа $\mathbf{v}_{j}^{r}$. Для каждого $i \in I$ справедливо равенство $a_{i}=v_{\gamma(i)}=k+1$, поскольку $\gamma(i) \neq j$. Таким образом, $\left.\mathbf{a}\right|_{I}=(k+1, \ldots, k+1)$.

Лемма 2. Пусть $I \subseteq N_{n}-$ индексируемое множество координат $n$-местного отношения $\rho$ с индексачией $\gamma$. Тогда любое $J \subseteq I$ индексируемо отображением $\gamma^{\prime}=\left.\gamma\right|_{J}$

\section{4. Кодирующие отношения}

Пусть $\rho$ - отношение на множестве $E_{k}, r-$ натуральное число и $s=\operatorname{ar} \rho$. Для $\mathbf{a} \in \rho$ через $\mathbf{w}_{\mathbf{a}}^{r}$ обозначим кортеж $\left(w_{1}, \ldots, w_{s p^{r}}\right)$, где $w_{i p^{r}+1}=\ldots=w_{(i+1) p^{r}}=a_{i}$. Обозначим через $\mathbf{v}^{\prime r}\left(\mathbf{s}^{\prime r}, \mathbf{t}^{\prime r}\right)$ кортеж такой, что для каждого $i \in N_{s}$ выполнено равенство $\left.\mathbf{v}^{\prime r}\right|_{\left\{i p^{r}+1, \ldots,(i+1) p^{r}\right\}}=\mathbf{v}_{j}^{r}$ (аналогично определяются $\mathbf{s}_{j}^{\prime r}, \mathbf{t}^{\prime r}$ ). Отношение $\varkappa_{\rho}^{r}$ такое, что $\mathbf{b} \in \varkappa_{\rho}^{r}$ тогда и только тогда, когда либо множество

$$
\left\{j \in\left\{0, \ldots, p^{r}-1\right\} \mid \text { найдется } i \text { такое, что } b_{i p^{r}+j} \neq k+2\right\}
$$

есть собственное подмножество в $\left\{0, \ldots, p^{r}-1\right\}$, либо $\mathbf{b}=\mathbf{t}^{\prime r}$, либо $\mathbf{b}=\mathbf{v}_{l}^{\prime r}, \mathbf{s}_{l}^{\prime r}$ для некоторого $l$, либо $\mathbf{b}=\mathbf{w}_{\mathbf{a}}^{r}$, где $\mathbf{a} \in \rho$.

Лемма 3. Для любых $\rho_{1}, \rho_{2} \in R_{k}$, где ar $\rho_{1}=m$, ar $\rho_{2}=n$, справедливы соотношенนя

$$
\begin{aligned}
\nabla_{p^{r}-1, p^{r}}\left(\ldots \nabla_{2, p^{r}}\left(\nabla_{1, p^{r}} \varkappa_{\rho_{1}}^{r}\right) \ldots\right) & =\varkappa_{\nabla_{1,2} \rho_{1}}^{r}, \\
\left(\varkappa_{\rho_{1}}^{r} \times \varkappa_{\rho_{2}}^{r}\right) \cap \varkappa_{v_{m+n}}^{r} & =\varkappa_{\rho_{1} \times \rho_{2}}^{r}, \\
\left.\varkappa_{\rho_{1}}^{r}\right|_{\left\{1, \ldots,(m-1) p^{r}\right\}} & =\varkappa_{\rho^{\prime}}^{r}, \quad \rho^{\prime}=\left.\rho_{1}\right|_{\{1, \ldots, m-1\}}, \\
\pi^{\prime} \varkappa_{\rho_{1}}^{r} & =\varkappa_{\pi \rho_{1}}^{r},
\end{aligned}
$$

әде $\pi$ - перестановка множества $N_{m}, \pi^{\prime}$ - перестановка множества $N_{p^{r} m}$, причем $\pi^{\prime}\left(i p^{r}+j\right)=\pi(i) p^{r}+j$ для всех $i \in N_{m} u j \in\left\{0, \ldots, p^{r}-1\right\}$.

Непосредственно из определения вытекает следующее утверждение. 
Лемма 4. Для любого $\rho \in R_{E_{k}}^{(n)}$, любого $r \in \mathbf{N} u I \subseteq N_{n p^{r}}$, если

$$
\left\{j \in\left\{0, \ldots, p^{r}-1\right\} \mid j \equiv i \quad\left(\bmod p^{r}\right) \text { для некоторого } \in I\right\} \neq\left\{0, \ldots, p^{r}-1\right\},
$$

то $\left.\varkappa_{\rho}^{r}\right|_{I}$ является универсалъным отношением.

\section{5. Описание индексаций кодирующих отношений}

В этом пункте мы полностью опишем индексации каждого подмножества множества координат кодирующего отношения. Это описание полезно по двум причинам. Вопервых, мы будем использовать его в дальнейших рассуждениях, а во-вторых, оно покажет, что кодирующие отношения имеют очень мало индексаций, а индексацит множества всех координат единственна, и поэтому мы можем с ее помощью отделят ь координаты одну от другой.

Отношение $\rho, \operatorname{ar} \rho=n$, назовем однозначно индексируемым, если оно имеет единственную индексацию.

Предложение 2. Для любого $\rho \in R_{k}$ и любого $r$ отношение $\varkappa_{\rho}^{r}$ однозначно индексируемо и его индексация $\gamma$ определяется сравнением $\gamma(i) \equiv i\left(\bmod p^{r}\right)$.

Доказательство. Пусть ar $\rho=s$. Рассмотрим ограничение $\gamma$ индексации множества $N_{s p^{r}}$ на множество $N_{p^{r}}$. Это отображение, очевидно, является индексацией. Пусть она имеет ранг $l$, т.е. $\gamma: N_{p^{r}} \rightarrow N_{p^{l}}$. Отображение $\gamma$ сюръективно, так как в противном случае по лемме 1 отношение $\left.\varkappa_{\rho}^{r}\right|_{N_{p}}$ содержало бы кортеж $(k+1, \ldots, k+1)$. Отсюда, $l \leqslant r$.

Кортеж, образ которого при отображении $\gamma$ является $j$-м выделяющим ( $j$-м связывающим, титульным, кодирующим) будем называть $j$-м $\gamma$-выделяющим $(j$-м $\gamma$ связывающим, $\gamma$-титульным, $\gamma$-кодирующим). Таким образом, один и тот же кортеж может быть одновременно, например, $\gamma$-выделяющим и $\gamma$-связывающим. Заметим еще, что проекциями кортежей $\mathbf{v}_{j}^{\prime r}, \mathbf{s}_{j}^{\prime r}, \mathbf{t}^{\prime r}$ на $N_{p^{r}}$ служат кортежи $\mathbf{v}_{j}^{r}, \mathbf{s}_{j}^{r}, \mathbf{t}^{r}$ соответственно.

Далее доказательство разобьем на 6 шагов.

На первом шаге докажем, что титульным кортежем для индексации $\gamma$ является кортеж $\mathbf{t}^{r}$. В самом деле, $\gamma$-титульный кортеж а содержит среди своих компонент лишь $k$ и $k+1$, поэтому он совпадает с одним из кортежей $\mathbf{v}_{j}^{r}, \mathbf{s}_{j}^{r}, \mathbf{t}^{r}$. Однако, $\left|\gamma^{-1}(\{1,2,3\})\right| \geqslant 3$ и $a_{i}=k$ для всех $i \in \gamma^{-1}(\{1,2,3\})$, поэтому а не может быть выделяющим или связывающим.

На втором шаге докажем, что выделяющими и связывающими кортежами для индексации $\gamma$ служат кортежи $\mathbf{v}_{j}^{r}$ и $\mathbf{s}_{j}^{r}$. Действительно, $\gamma$-выделяющими и $\gamma$-связывающими могут быть лишь кортежи вида $\mathbf{v}_{j}^{r}, \mathbf{s}_{j}^{r}, \mathbf{t}^{r}$, поскольку все прочие кортежи содержат компоненты не равные $k, k+1$. Далее, поскольку $\gamma$ сюръективно, прообразы различных кортежей различны. Так как $\mathbf{t}^{r} \gamma$-титульный, он не может быть равен никакому $\gamma$-выделяющему или $\gamma$-связывающему кортежу.

На третьем шаге докажем, что $\gamma$ биективно. Предположим, что $\gamma\left(i_{1}\right)=\gamma\left(i_{2}\right)=j$ для некоторых $i_{1}, i_{2} \in N_{p^{r}}$. Рассмотрим $j$-й $\gamma$-связывающий кортеж а (если $j=p^{r}$, то нужно рассмотреть $\gamma$-связывающий кортеж с номером $\left.p^{r}-1\right)$. Этот кортеж имеет по крайней мере три равные $k$ компоненты $a_{i_{1}}=a_{i_{2}}=a_{i}=k$, где $i-$ какой-нибудь элемент из прообраза $j+1$. Но в силу второго шага кортеж а не содержится в $\left.\varkappa_{\rho}^{r}\right|_{N_{p} r}$.

Таким образом, $\gamma$-выделяющими кортежами являются кортежи вида $\mathbf{v}_{j}^{r}$, a $\gamma$ связывающими - кортежи вида $\mathbf{s}_{j}^{r}$. 
На четвертом шаге докажем, что для любого $i \in N_{p^{r-1}}$ справедливо включение $\gamma(i+1) \in\{\gamma(i)-1, \ell \gamma(i)+1\}$. Пусть $\gamma(i)=j$. Рассмотрим $(j-1)$-й и $j$-й $\gamma$-связывающие кортежи a, b. Справедливы равенства $a_{i}=b_{i}=k$, а также $a_{\gamma^{-1}(j-1)}=b_{\gamma^{-1}(j+1)}=k$. Отсюда, $\mathbf{a}, \mathbf{b} \in\left\{\mathbf{s}_{i}^{r}, \mathbf{s}_{i-1}^{r}\right\}$, а так как $\gamma$ биективно,

$$
\{j-1, j+1\}=\{\gamma(i-1), \gamma(i+1)\}
$$

что и требовалось доказать. В случае, когда $j=1$ или $j=p^{r}$, достаточно рассматривать лишь один связывающий кортеж.

На пятом шаге установим равенство $\gamma(1)=1$. Пусть $\mathbf{b}-$ образ $\mathbf{t}^{r}$, тогда $b_{\gamma(1)}=k$. Заметим, что поскольку образ $\mathbf{t}^{r}$ есть связывающий кортеж, то $\gamma(1) \in\{1,2,3\}$. Пусть $\gamma(1)=j, j \neq 1$ и $\gamma(2)=j+1$. Рассмотрим $(j-1)$-й $\gamma$-связывающий кортеж а. Справедливы равенства $a_{1}=a_{\gamma^{-1}(j)}=k$. В $\left.\varkappa_{\rho}^{r}\right|_{N_{p^{r}}}$ есть лишь один связывающий кортеж с таким свойством - это $\mathrm{s}_{1}^{r}$. Но тогда $a_{2}=k$, и потому

$$
\gamma^{-1}(j-1)=2=\gamma^{-1}(j+1)
$$

что противоречит биективности $\gamma$. Случай $\gamma(2)=j-1$ рассматривается аналогично.

Наконец, на шестом шаге докажем, что $\gamma$ тождественно. На предыдущем шаге установлено, что $\gamma(1)=1$. Далее, $\gamma(2) \in\{\gamma(1)+1, \gamma(1)-1\}$, но случай $\gamma(1+1)=$ $\gamma(1)-1$ невозможен, значит, $\gamma(2)=2$. Предположим, что мы доказали равенство $\gamma(i)=i$ для всех $i \leqslant j$. Рассмотрим $\gamma(j+1)$. Согласно шагу $4 \gamma(j+1)=\gamma(j)+1=j+1$, либо $\gamma(j+1)=\gamma(j)-1=j-1$. Но в последнем случае $\gamma(j-1)=j-1=\gamma(j+1)$, что противоречит биективности $\gamma$. Таким образом, мы получаем, что $\gamma$ - тождественное отображение.

Аналогично проверяется, что для любого $i \in N_{s}$ ограничение индексации на множество $\left\{i p^{r}+1, \ldots,(i+1) p^{r}\right\}$ есть отображение, переводящее $i p^{r}+j$ в $j$. Таким образом, каждая индексация отношения $\varkappa_{\rho}^{r}$ имеет вид, указанный в формулировке предложения. Предложение 2 доказано.

Если же множество $J \subseteq N_{p^{r}}$ не равно $N_{p^{r}}$, то любое отображение множества $I$ в $N_{p^{m}}$ для любого $m$ является $m$-индексацией, поскольку $\left.\varkappa_{\rho}^{r}\right|_{I}-$ универсальное отношение в силу леммы 4.

Пусть $J=\left\{i_{1} p^{r}+1, \ldots, i_{p^{r}} p^{r}+p^{r}\right\}$, где $i_{1}, \ldots, i_{p^{r}} \in N_{\text {ar } \rho}$. Тогда, очевидно, отношения $\left.\varkappa_{\rho}^{r}\right|_{J}$ и $\left.\varkappa_{\rho}^{r}\right|_{\left\{1, \ldots, p^{r}\right\}}$ отличаются лишь кортежами, чьи компоненты содержатся в множестве $E_{k}$.

Следствие 2. Если множество $J$ координат отношения $\varkappa_{\rho}^{r}$ таково, ито

$$
\left\{i \in N_{p^{r}} \mid j \equiv i \quad\left(\bmod p^{r}\right), j \in J\right\}=N_{p^{r}}
$$

то $J$ имеет единственную индексачию, которая определена в предложении 2 . $B$ противном случае, любое отображение $\gamma: J \rightarrow N_{p^{m}}$ является индексацией $J$. 


\section{5. Построение вложения}

\section{1. Множество отношений $\mathrm{SN}$}

Пусть $\rho-n$-местное отношение. Положим $M_{\rho}: \mathscr{P}\left(N_{n}\right) \rightarrow \mathscr{P}\left(N_{n}\right)$ - отображение множества подмножеств из $N_{n}$ в себя такое, что

$$
\begin{gathered}
M_{\tau}(I)=\left\{j \in N_{n} \mid \text { найдется } j \in I \text { такое, что для всех } \mathbf{a} \in \tau\right. \\
\text { из } \left.\left\{a_{i} \mid i \in I\right\} \subseteq E_{k+2} \text { следует, что } a_{j} \in E_{k+2}\right\} .
\end{gathered}
$$

Нетрудно проверить, что отображение $M_{\tau}$ является оператором замыкания. Замкнутые для этого оператора множества мы будем называть замкнутыми множествами координат. Таким образом, множество $I$ замкнуто тогда и только тогда, когда для всякого $j \notin I$ найдется кортеж $\mathbf{a} \in \tau$ такой, что $a_{i} \in E_{k+2}$ для всех $i \in I$ и $a_{j}=k+2$. Пусть также $\sigma(\tau)$ - отношение эквивалентности на множестве $N_{n}$, определенное следующим образом: $(i, j) \in \sigma(\tau)$ тогда и только тогда, когда $a_{i}=a_{j}$ для всех $\mathbf{a} \in \tau$. Как обычно, через $i^{\sigma(\tau)}\left(I^{\sigma(\tau)}\right)$ обозначается класс эквивалентности, содержащий $i \in N_{n}$ (объединение классов, содержащих элементы из $I \subseteq N_{n}$ ). Для каждого отношения $\rho$ найдется отношение $\rho^{\prime}$ такое, что $\sigma\left(\rho^{\prime}\right)$ есть отношение равенства и $\rho$ может быть получено из $\rho^{\prime}$ удвоениями координат, а $\rho^{\prime}$ из $\rho$ отождествлениями. Поэтому всюду, кроме основных определений, мы предполагаем, что $\sigma(\rho)$ - отношение равенства.

Для произвольного $I \subseteq N_{n}$ через $\xi(\tau, I)$ обозначим отношение эквивалентности на $I$, определенное следующим образом:

$$
\xi(\tau, I)=\left\{(i, j) \in I^{2} \mid a_{i}=a_{j} \text { для всех } \mathbf{a} \in \tau \text { таких, что }\left.\mathbf{a}\right|_{I} \in E_{k}^{|I|}\right\} .
$$

Предположим теперь, что $I-$ индексируемое множество и $\left\{i_{1}, \ldots, i_{s}\right\}-$ некоторый набор представителей классов эквивалентности $\xi(\tau, I)$. Положим

$$
K(\tau, I)=\left.\left(\left.\tau\right|_{I} \cap E_{k}^{|I|}\right)\right|_{\left\{i_{1}, \ldots, i_{s}\right\}}
$$

Аналогично, для $\mathbf{a}=\left(a_{i}\right)_{i \in I}$ такого, что $\mathbf{a} \in E_{k}^{|I|}$ и $a_{i}=a_{j}$ для всех $(i, j) \in \xi(\rho, I)$, обозначим $K(\mathbf{a})=\left.\mathbf{a}\right|_{\left\{i_{1}, \ldots, i_{s}\right\}}$. Таким образом, $K\left(\varkappa_{\rho}^{r}, N_{\operatorname{ar}} p^{r}\right)=\rho$ и $K\left(\mathbf{w}_{\mathbf{a}}^{r}\right)=\mathbf{a}$.

Для $n$-местного кортежа а через $2_{I}(\mathbf{a})$, где $I \subseteq N_{n}$, обозначим кортеж $\left(b_{1}, \ldots, b_{n}\right)$, для которого $b_{i}=a_{i}$ при $i \in I$ и $b_{i}=k+2$ в противном случае. Кортеж вида $(k+1, \ldots, k+1)$ будем называть единичным. Через SN обозначим множество отношений $\tau$, удовлетворяющих следующим условиям.

SN1. Для всякого $I \subseteq N_{n}$ и $\mathbf{a} \in \tau$ кортеж $2_{M_{\tau}(I)}(\mathbf{a})$ содержится в $\tau$.

$\mathrm{SN2}$. Если $\left.\tau\right|_{I}$ содержит единичный кортеж, то $\left.\tau\right|_{I}-$ тривиальное отношение и $M(I)=I^{\sigma(\tau)}$.

SN3. Если для $I$ найдутся индексации $\gamma, \gamma^{\prime}$ различного ранга, то $\left.\tau\right|_{I}-$ тривиальное отношение.

SN4. Если для $I$ найдутся две индексации $\gamma, \gamma^{\prime}$ одинакового ранга и $\gamma(i) \neq \gamma^{\prime}(i)$ для некоторого $i \in I$, то

$$
\left.\tau\right|_{I^{\sigma(\rho)}}=\left.\tau\right|_{I^{\sigma(\tau)} \backslash\left\{i^{\sigma(\tau)}\right\}} \times\left.\tau\right|_{\left\{i^{\sigma(\tau)}\right\}}
$$


SN5. Для каждой индексации множества $I$ найдется индексация множества $M_{\tau}(I)$, причем для всякого $\mathbf{a} \in \tau$ из того, что $\left.\mathbf{a}\right|_{I}-$ кодирующий кортеж, следует, что и $\left.\mathbf{a}\right|_{M_{\tau}(I)}$ - кодирующий кортеж, если $\left.\mathbf{a}\right|_{I}-$ выделяющий (связывающий, титульный) кортеж с номером $j$, то $\left.\mathbf{a}\right|_{M_{\tau}(I)}$ - выделяющий (связывающий, титульный) кортеж с тем же номером.

SN6. Если $I$ - индексируемое множество, то $K(\tau, I)$ является тривиальным отношением.

Как показывает следующая лемма, множество SN непусто.

Лемма 5. Для любого $r$ июбого $\rho \in R_{k}$ отношение $\varkappa_{\rho}^{r}$ удовлетворяет условиям SN1-SN5.

Доказательство. Введем обозначения $\tau=\varkappa_{\rho}^{r}$, ar $\rho=s, n=s p^{r}$, и пусть $\gamma_{0}-$ (единственная) индексация отношения $\tau$. Для $I \subseteq N_{n}$ положим $S_{I}=\gamma_{0}(I)$. Заметим сначала, что

$$
M_{\tau}(I)= \begin{cases}I, & \text { если } S_{I} \neq N_{p^{r}}, \\ N_{n} & \text { в противном случае. }\end{cases}
$$

Таким образом, условие SN1 непосредственно вытекает из определения кодирующего отношения.

Если $\left.\tau\right|_{I}$ содержит единичный кортеж, то $S_{I} \neq N_{p^{r}}$, а потому $\left.\tau\right|_{I}$ универсально, то есть SN2 также выполнено.

Далее, для любого $I$ либо $\left.\tau\right|_{I}$ универсально, либо имеет единственную индексацию согласно предложению 2. Следовательно, SN3 и SN4 верны.

Проверим SN5. Если $S_{I} \neq N_{p^{r}}$, то $M_{\tau}(I)=I$ и условие выполнено. В противном случае, если $\mathbf{a} \in \tau$ и $\left.\mathbf{a}\right|_{I}$ - выделяющий (связывающий, титульный или кодирующий) кортеж, то и весь а таков.

Для любого $I \subseteq N_{s p^{r}}$ проекция $\left.\varkappa_{\rho}^{r}\right|_{I}$, где $s=\operatorname{ar} \rho$, либо является универсальным отношением, либо $K\left(\left.\tau\right|_{I}\right)$ является проекцией $\rho$ на множество

$$
J=\left\{j \in N_{s} \mid \text { найдется } i \in N_{p^{r}} \text { такой, что }(j-1) p^{r}+i \in I\right\} .
$$

Лемма 5 влечет следующее утверждение.

Следствие 3. Для любого $I \subseteq N_{n}$, где $n=\operatorname{ar} \varkappa_{\rho}^{r}$, отношение $K\left(\varkappa_{\rho}^{r}, I\right)$ является проекцией отношения $\rho$.

Таким образом, если $\rho$ тривиально, то $K\left(\left.\tau\right|_{I}\right)$ также тривиально.

Следствие 4. Для любого тривиального отношения $\rho \in R_{k}$ и любого $r$ отношение $\varkappa_{\rho}^{r}$ содержится в $\mathrm{SN}$.

Пусть $r \in \mathbf{N}$ и $C-$ клон отношений на $E_{k}$. Скажем, что $\tau$ удовлетворяет условию $\mathrm{SN}(r, C)$, если для любого $I \subseteq \operatorname{ar} \tau$, для которого найдется индексация ранга $r$, справедливо включение $K(\tau, I) \in C$.

Лемма 6. Операции клона отношений сохраняют свойства $\mathrm{SN} 1-\mathrm{SN} 5$ u $\mathrm{SN}(r, C)$ для любых $r \in \mathbf{N}$ и клона отношений $C$. 
Доказательство. Нужно проверить, что проекции, прямое произведение и отождествление координат сохраняют $\mathrm{SN} 1-\mathrm{SN} 5$ и $\mathrm{SN}(r, C)$.

Докажем, что проекции сохраняют эти свойства. Пусть $\tau$ удовлетворяет SN1-SN5 и $\mathrm{SN}(r, C)$ для некоторых $r \in \mathbf{N}$ и $C, J \subseteq N_{n}$, где $n=\operatorname{ar} \tau$, и $\tau^{\prime}=\left.\tau\right|_{J}$. Возьмем $I \subseteq J$. Очевидно, $M_{\tau^{\prime}}(I)=M_{\tau}(I) \cap J$ и $\left.\tau^{\prime}\right|_{I}=\left.\tau\right|_{I}$. Отсюда, по свойству SN1 отношения $\tau$ немедленно следует, что

$$
2_{M_{\tau^{\prime}}(I)}(\mathrm{a})=\left.2_{M_{\tau}(I)}\left(\mathbf{a}^{\prime}\right)\right|_{J} \in \tau^{\prime}
$$

для любого $\mathbf{a}^{\prime} \in \tau$ и $\mathbf{a}=\left.\mathbf{a}^{\prime}\right|_{I} \in \tau^{\prime}$. Таким образом, SN1 выполнено. Если $\left.\tau^{\prime}\right|_{I}$ содержит единичный кортеж, то

$$
M_{\tau^{\prime}}(I)=M_{\tau}(I) \cap J=I \cap J=I
$$

и $\left.\tau^{\prime}\right|_{I}=\left.\tau\right|_{I}$ является универсальным отношением.

Если $\gamma_{1}, \gamma_{2}$ - индексации $I$ различных рангов, то $\left.\tau^{\prime}\right|_{I}=\left.\tau\right|_{I}$ есть тривиальное отношение, что доказывает SN3. Пусть $\gamma_{1}, \gamma_{2}$ - индексации множества I отношения $\tau^{\prime}$ такие, что $\gamma_{1}(i) \neq \gamma_{2}(i)$ для некоторого $i \in I$. Тогда по SN4 для $\tau$

$$
\left.\tau^{\prime}\right|_{I}=\left.\tau\right|_{I}=\left.\tau\right|_{I \backslash\{i\}} \times\left.\tau\right|_{\{i\}}=\left.\tau^{\prime}\right|_{I \backslash\{i\}} \times\left.\tau^{\prime}\right|_{\{i\}} .
$$

Свойство SN5 отношения $\tau^{\prime}$ непосредственно вытекает из SN5 для $\tau$, а сохранение $\mathrm{SN}(r, C)$ следует из равенства $K\left(\tau^{\prime}, I\right)=K(\tau, I)$, так как $\left.\tau^{\prime}\right|_{I}=\left.\tau\right|_{I}$.

Докажем, что операция взятия прямого произведения сохраняет свойства SN1$\mathrm{SN} 5$ и $\mathrm{SN}(r, C)$. Пусть $\tau=\tau_{1} \times \tau_{2}, \tau_{1}, \tau_{2}$ удовлетворяют $\mathrm{SN} 1-\mathrm{SN} 5, \mathrm{SN}(r, C)$ для некоторых $r \in \mathbf{N}$ и $C, J_{1}, J_{2}-$ множества координат отношений $\tau_{1}, \tau_{2}$ (при этом мы полагаем $\left.J_{1} \cap J_{2}=\varnothing\right), I \subseteq J_{1} \cup J_{2}$ и $I^{1}=I \cap J_{1}, I^{2}=I \cap J_{2}$. Очевидно равенство

$$
M_{\tau}(I)=M_{\tau_{1}}\left(I^{1}\right) \cup M_{\tau_{2}}\left(I^{2}\right) .
$$

Свойство SN1 следует из равенства

$$
2_{M_{\tau}(I)}(\mathbf{a})=2_{M_{\tau_{1}}\left(I^{1}\right)}\left(\left.\mathbf{a}\right|_{J_{1}}\right) \times 2_{M_{\tau_{2}}\left(I^{2}\right)}\left(\left.\mathbf{a}\right|_{J_{2}}\right) .
$$

Если $\left.\tau\right|_{I}$ содержит единичный кортеж, то $\left.\tau_{1}\right|_{I^{1}},\left.\tau_{2}\right|_{I^{2}}$ также содержат единичный кортеж. Отсюда,

$$
M_{\tau}(I)=M_{\tau_{1}}\left(I^{1}\right) \cup M_{\tau_{2}}\left(I^{2}\right)=I^{1} \cup I^{2}=I,\left.\quad \tau_{1}\right|_{I^{1}},\left.\quad \tau_{2}\right|_{I^{2}}
$$

универсальны, а потому $\left.\tau\right|_{I}=\left.\tau_{1}\right|_{I^{1}} \times\left.\tau_{2}\right|_{I^{2}}$ также универсально. Таким образом, SN2 выполнено.

Если $\gamma_{1}, \gamma_{2}$ - индексации множества $I$, имеющие различные ранги, то $\left.\gamma_{1}\right|_{I^{1}},\left.\gamma_{2}\right|_{I^{1}}$ и $\left.\gamma_{1}\right|_{I^{2}},\left.\gamma_{2}\right|_{I^{2}}$ также имеют различные ранги. Поэтому $\left.\tau_{1}\right|_{I^{1}},\left.\tau_{2}\right|_{I^{2}}$ тривиальны, а значит, $\left.\rho\right|_{I}=\left.\tau_{1}\right|_{I^{1}} \times\left.\tau_{2}\right|_{I^{2}}$ тривиально.

Допустим, что $\gamma_{1}, \gamma_{2}$ - индексации множества $I$, удовлетворяющие посылке условия SN4, то есть $\gamma_{1}(i) \neq \gamma_{2}(i)$. Без ограничения общности можно считать, что $i \in I_{1}$. Отсюда,

$$
\left.\tau_{1}\right|_{I^{1}}=\left.\tau_{1}\right|_{I^{1} \backslash\{i\}} \times\left.\tau\right|_{\{i\}}
$$

по свойству SN4. Таким образом,

$$
\left.\tau\right|_{I}=\left.\tau_{1}\right|_{I^{1} \backslash\{i\}} \times\left.\tau\right|_{\{i\}} \times\left.\tau\right|_{I^{2}}=\left.\tau\right|_{I \backslash\{i\}} \times\left.\tau\right|_{\{i\}},
$$


что и требовалось доказать.

Требуемым в условии SN5 продолжением индексации $\gamma$ множества $I$ на $M_{\tau}(I)$ служит отображение $\gamma^{\prime}$, которое на $M_{\tau_{j}}\left(I^{j}\right), j=1,2$, совпадает с существующим по SN5 для $\tau_{j}$ продолжением индексации $\left.\gamma\right|_{I^{j}}$. При этом, если $\left.\mathbf{a} \in \tau\right|_{M(I)}$ таков, что $\left.\mathbf{a}\right|_{I}-$ выделяющий (связывающий или титульный) кортеж, то $\left.\mathbf{a}\right|_{M_{\tau_{1}}\left(I^{1}\right)},\left.\mathbf{a}\right|_{M_{\tau_{2}}\left(I^{2}\right)}-$ выделяющие (связывающие, титульные) кортежи с тем же номером. Следовательно, и а таков.

Наконец, если $\mathbf{a}_{1} \in \tau_{1}, \mathbf{a}_{2} \in \tau_{2}-$ кодирующие кортежи, то $\mathbf{a}_{1} \times \mathbf{a}_{2}-$ также кодирующий кортеж и обратно. Далее,

$$
\xi(\tau, I)=\xi\left(\tau_{1}, I^{1}\right) \cup \xi\left(\tau_{2}, I^{2}\right)
$$

за исключением случая, когда найдутся $i_{1} \in I^{1}, i_{2} \in I^{2}$, входящие в соответствующие системы представителей и такие, что отношения $\left.K\left(\tau_{1}, I^{1}\right)\right|_{\left\{i_{1}\right\}}$ и $\left.K\left(\tau_{2}, I^{2}\right)\right|_{\left\{i_{2}\right\}}$ равны и одноэлементны. Таким образом,

$$
K(\tau, I)=K\left(\tau_{1}, I^{1}\right) \times K\left(\tau_{2}, I^{2}\right)
$$

В последнем же случае

$$
K(\tau, I)=\nabla_{i_{1}, i_{2}}\left(K\left(\tau_{1}, I^{1}\right) \times K\left(\tau_{2}, I^{2}\right)\right),
$$

если $i_{1}, i_{2}$ - единственная пара с таким свойством. Если таких пар более одной, то $K(\tau, I)$ получается в результате нескольких отождествлений координат. Итак, $K(\tau, I) \in C$, если $K\left(\tau_{1}, I^{1}\right), K\left(\tau_{2}, I^{2}\right) \in C$.

Покажем, что отождествление сохраняет свойства $\mathrm{SN} 1-\mathrm{SN} 5$ и $\mathrm{SN}(r, C)$. Пусть $\tau^{\prime}=\nabla_{n-1, n} \tau$ и $\tau$ удовлетворяет $\mathrm{SN} 1-\mathrm{SN} 5, \mathrm{SN}(r, C)$ для некоторых $r \in \mathrm{N}$ и $C$. Так как $\tau$ удовлетворяет $\mathrm{SN} 1$,

$$
M_{\tau^{\prime}}= \begin{cases}M_{\tau}(I), & \text { если } n-1 \notin M_{\tau}(I), \\ M_{\tau}(I \cup\{n-1, n\}) \backslash\{n\} & \text { в противном случае. }\end{cases}
$$

Поскольку каждое замкнутое для $\tau^{\prime}$ множество координат является также замкнутым для $\tau$ (с точностью до наличия $n$-й координаты), свойство SN1 отношения $\tau^{\prime}$ следует из соответствующего свойства для $\tau$.

Возьмем $I \subseteq N_{n-1}$ и положим $I^{\prime}=I$, если $n-1 \notin I$ и $I^{\prime}=I \cup\{n\}$ при $n-1 \in$ I. Если $\left.\tau^{\prime}\right|_{I}$ содержит единичный кортеж, то и $\left.\tau\right|_{I^{\prime}}$ его содержит. Отсюда, $\left.\tau\right|_{I^{\prime}}-$ универсальное отношение и $M_{\tau}\left(I^{\prime}\right)=I^{\prime}$. Таким образом, $M_{\tau^{\prime}}(I)=I$ и в силу SN1 отношения $\tau$ получаем, что $\left.\tau^{\prime}\right|_{I}=v_{|I|}$.

Прежде чем доказывать оставшиеся свойства, заметим, что если $\gamma$ - индексация множества $I$ и $n-1 \in I$, то отображение $\gamma^{\prime}$, где $\gamma^{\prime}(i)=\gamma(i)$ при $i \in I$ и $\gamma^{\prime}(n)=\gamma(n-1)$, является индексацией множества координат $I \cup\{n\}$ отношения $\tau$.

Пусть $\gamma_{1}, \gamma_{2}$ - индексации множества $I$, а $\gamma_{1}^{\prime}, \gamma_{2}^{\prime}$ - указанные в предыдущем абзаце индексации множества $I^{\prime}$. Если $\gamma_{1}, \gamma_{2}$ различного ранга, то и $\gamma_{1}^{\prime}, \gamma_{2}^{\prime}$ имеют различный ранг. Отсюда, $\left.\tau\right|_{I^{\prime}}$ тривиально, а потому и $\left.\tau^{\prime}\right|_{I}$ тривиально. Условие SN3 выполнено. Пусть теперь $\gamma_{1}, \gamma_{2}$ и $i \in I$ удовлетворяют посылке условия SN4. Для $\gamma_{1}^{\prime}, \gamma_{2}^{\prime}$ выполнены те же условия, а значит,

$$
\left.\tau\right|_{I^{\prime}}=\left.\tau\right|_{I^{\prime} \backslash\{i\}} \times\left.\tau\right|_{\{i\}} .
$$


Если $n \notin I^{\prime}$, то $\left.\tau^{\prime}\right|_{I}=\left.\tau\right|_{I^{\prime}}$ в силу SN1. Иначе, если $i \neq n-1$, то

$$
\begin{aligned}
\left.\tau^{\prime}\right|_{I} & =\left.\nabla_{n-1, n} \tau\right|_{I^{\prime}}=\nabla_{n-1, n}\left(\left.\tau\right|_{I^{\prime} \backslash\{i\}} \times\left.\tau\right|_{\{i\}}\right) \\
& =\nabla_{n-1, n}\left(\left.\tau\right|_{I^{\prime} \backslash\{i\}}\right) \times\left.\tau\right|_{\{i\}}=\left.\tau^{\prime}\right|_{I \backslash\{i\}} \times\left.\tau^{\prime}\right|_{\{i\}} .
\end{aligned}
$$

Пусть, наконец, $i=n-1$. Тогда $n-1$ и $n$ удовлетворяют посылке SN4 для индексаций $\gamma_{1}^{\prime}, \gamma_{2}^{\prime}$. Отсюда,

$$
\left.\tau\right|_{I^{\prime}}=\left.\tau\right|_{I^{\prime} \backslash\{n-1, n\}} \times\left.\tau\right|_{\{n-1\}} \times\left.\tau\right|_{\{n\}} .
$$

Таким образом,

$$
\left.\tau^{\prime}\right|_{I}=\left.\tau^{\prime}\right|_{I \backslash\{n-1\}} \times\left.\tau^{\prime}\right|_{\{n-1\}}
$$

Проверим SN5. Если $n-1, n \notin M_{\tau}(I)$, то SN5 следует из соответствующего свойства $\tau$. Предположим, $n-1 \in M_{\tau}(I), \gamma$ - индексация $I$ отношения $\tau^{\prime}$. Тогда отображение $\gamma$ является также индексацией множества координат $I$ отношения $\tau$. Пусть $\gamma^{\prime}$ - продолжение $\gamma$ на $M_{\tau}(I)$ согласно свойству SN5. Доопределим $\gamma^{\prime}$ на множестве $M_{\tau}(I) \cup\{n\}$ как указано выше, получим индексацию $\gamma^{\prime \prime}$. Далее, вновь по свойству SN5 отношения $\tau$ существует продолжение $\gamma^{\prime \prime \prime}$ отображения $\gamma^{\prime \prime}$ на

$$
M_{\tau}\left(M_{\tau}(I) \cup\{n\}\right)=M_{\tau}(I \cup\{n\}) .
$$

Пусть $\mathbf{a} \in \tau$ таков, что $\left.\mathbf{a}\right|_{I}$ - выделяющий (связывающий, титульный) кортеж и $a_{n-1}=a_{n}$. Такой кортеж найдется, так как $\left.\left.\mathbf{a}\right|_{I} \in \tau^{\prime}\right|_{I}$ по определению индексации. Тогда согласно SN5

$$
\left.\left.\mathbf{a}\right|_{M_{\tau}(I \cup\{n\})} \in \tau\right|_{M_{\tau}(I \cup\{n\})}
$$

- выделяющий (связывающий, титульный) кортеж с тем же номером для $\gamma^{\prime \prime \prime}$. Так как $a_{n-1}=a_{n}$, справедливо включение $\left.\mathbf{a}\right|_{N_{n-1}} \in \tau^{\prime}$, то есть ограничение $\gamma^{\prime \prime \prime}$ на $M_{\tau^{\prime}}(I)$ является индексацией.

Пусть, наконец, $\gamma$ - индексация множества $I, \gamma^{\prime}$ - соответствующая ей индексация $I^{\prime}$. Достаточно рассматривать случай, когда $I$ замкнуто и $n-1 \in I$. Если $n-1, n$ содержатся в одном классе эквивалентности $\xi(\tau, I)$, то $a_{n-1}=a_{n}$ всякий раз, когда $\left.\mathbf{a}\right|_{I^{\prime}} \in E_{k}^{\left|I^{\prime}\right|}$. Отсюда,

$$
K\left(\left.\tau^{\prime}\right|_{I}\right)=K\left(\left.\nabla_{n-1, n} \tau\right|_{I^{\prime}}\right)=K\left(\left.\tau\right|_{I^{\prime}}\right)
$$

Если же $n-1$ содержится в $s$-м, а $n$ в $t$-м классах эквивалентности $\xi\left(\tau, I^{\prime}\right)$, то для каждого $\left.\mathbf{a} \in \tau\right|_{I^{\prime}} \cap E_{k}^{\left|I^{\prime}\right|}$ такого, что $a_{n-1}=a_{n}$, справедливо равенство $a_{i}=a_{j}$, где $i, j$ содержатся в объединении $s$-го и $t$-го классов $\xi(\tau, I)$. Каждый класс $\xi\left(\tau^{\prime}, I\right)$ есть объединение классов эквивалентности $\xi\left(\tau, I^{\prime}\right)$ (с точностью до наличия $n$ ). Далее, нетрудно видеть, что кортеж $\mathbf{a}=\left(a_{1}, \ldots, a_{n-1}\right)$, для которого $\left.\mathbf{a}\right|_{I} \in E_{k}^{|I|}, a_{i}=a_{j}$ всякий раз, когда $(i, j) \in \xi\left(\tau^{\prime}, I\right)$ и $\left(a_{1}, \ldots, a_{n-1}, a_{n-1}\right) \in \tau$, содержится в $\tau^{\prime}$. Отсюда, $K\left(\tau^{\prime}, I\right)$ получено из $\nabla_{s, t} K\left(\tau, I^{\prime}\right)$ отождествлениями тех координат $i, j$, для которых $\mathbf{b} \in \nabla_{s, t} K\left(\tau, I^{\prime}\right)$ влечет $b_{i}=b_{j}$. Таким образом, $K\left(\tau^{\prime}, I\right) \in C$, если $K\left(\tau, I^{\prime}\right) \in C$.

Лемма 6 доказана.

\section{Следствие 5. Множество отношений SN является клоном отношений.}

Искомое вложение $\psi$ определим следующим образом. Пусть

$$
\mathbf{C}=\left(C_{1}, \ldots, C_{n}, \ldots\right)
$$


- последовательность клонов отношений из $R_{k}$ Последовательности $\mathbf{C}$ поставим в соответствие клон отношений

$$
\psi(\mathbf{C})=\left\langle\mathrm{SN} \cup\left\{\varkappa_{\rho}^{r} \mid \rho \in C_{r}, r \in \mathbf{N}\right\}\right\rangle .
$$

Из леммы 3 непосредственно следует, что

$$
\psi\left(\mathbf{C}^{1} \vee \mathbf{C}^{2}\right) \subseteq \psi\left(\mathbf{C}^{1}\right) \vee \psi\left(\mathbf{C}^{2}\right)
$$

для любых последовательностей клонов $\mathbf{C}^{1}, \mathbf{C}^{2}$, а значит, отображение $\psi$ сохраняет решеточные объединения. Далее, поскольку

$$
\mathrm{SN} \cup\left\{\varkappa_{\rho}^{r} \mid \rho \in \in C_{r}, r \in \mathbf{N}\right\}
$$

удовлетворяет всем условиям $\mathrm{SN}\left(r, C_{r}\right)$, то по лемме $6 \psi(\mathbf{C})$ также им удовлетворяет. Поэтому $\psi$ взаимно однозначно.

Таким образом, нам необходимо проверить, что $\psi$ сохраняет пересечения и отображает $\mathscr{L}_{k}^{\partial} \times \ldots \mathscr{L}_{k}^{\partial} \times \ldots$ на интервал решетки $\mathscr{L}_{k+3}^{\partial}$.

\section{6. Строение суперпозиций отношений из $\psi(\mathbf{C})$}

Пусть отношение $\tau$ определено формулой

$$
P_{\tau}\left(x_{1}, \ldots, x_{n}\right)=\exists\left(x_{i}\right)_{i \in\{n+1, \ldots, m\}} \bigwedge_{\alpha \in \Lambda} P_{\tau_{\alpha}}\left(x_{\pi_{\alpha}(i)}\right)_{i \in N_{n_{\alpha}}}
$$

где

$$
\tau_{\alpha} \in \mathrm{SN} \cup\left\{\varkappa_{\rho}^{r} \mid \rho \in R_{k}, r \in \mathbf{N}\right\},
$$

$n_{\alpha}=\operatorname{ar} \tau_{\alpha}$ и $\pi_{\alpha}: N_{n_{\alpha}} \rightarrow N_{m}(\alpha \in \Lambda)$. Пусть

$$
P_{\bar{\tau}}\left(x_{1}, \ldots, x_{m}\right)=\bigwedge_{\alpha \in \Lambda} P_{\tau_{\alpha}^{\prime}}\left(x_{\pi_{\alpha}(i)}\right)_{i \in N_{n_{\alpha}}}
$$

где $\tau_{\alpha}^{\prime}=\tau_{\alpha}$, если $\tau_{\alpha} \in \mathrm{SN}$, и $\tau_{\alpha}^{\prime}=\varkappa_{v}^{r}$, если $\tau_{\alpha}=\varkappa_{\rho}^{r}$, причем $\operatorname{ar} v=\operatorname{ar} \rho$ и $v$ есть универсальное отношение. Рассмотрим отношение $\tau^{\prime}$, задаваемое формулой

$$
P_{\tau^{\prime}}\left(x_{1}, \ldots, x_{n}\right)=\exists\left(x_{i}\right)_{i \in\{n+1, \ldots, m\}} P_{\bar{\tau}} .
$$

Согласно следствиям 4, 5 справедливо включение $\tau^{\prime} \in \mathrm{SN}$.

Лемма 7. Для любого $I \subseteq N_{n}$ справедливо равенство $M_{\tau}(I)=M_{\tau^{\prime}}(I)$.

Доказательство. Достаточно проверить, что $\tau$ и $\tau^{\prime}$ имеют одни и те же замкнутые множества. Поскольку $\tau_{\alpha} \subseteq \tau_{\alpha}^{\prime}$ для всех $\alpha \in \Lambda$, то $\tau \subseteq \tau^{\prime}$. Поэтому каждое множество, замкнутое для $\tau$, замкнуто для $\tau^{\prime}$.

Пусть теперь $I=M_{\tau^{\prime}}(I)$. Проверим, что для всякого а $\in \tau$ кортеж $2_{I}(\mathbf{a})$ содержится в $\tau$. Пусть $\mathbf{b}$ допустим для (2) и $\left.\mathbf{b}\right|_{N_{n}}=\mathbf{a}$. Тогда $\mathbf{b} \in \bar{\tau}$ и $\mathbf{c}=2_{M_{*}(I)}(\mathbf{b}) \in \bar{\tau}$, поскольку $\bar{\tau} \in \mathrm{SN}$ по следствию 5. Кортеж с допустим для (2). Действительно, если $\tau_{\alpha}$ не является кодирующим, то $\tau_{\alpha}^{\prime}=\tau_{\alpha}$ и $\left(c_{\pi_{\alpha}(i)}\right)_{i \in N_{n_{\alpha}}} \in \tau_{\alpha}$. Далее, если $\tau_{\alpha}$ кодирующее и среди $c_{\pi_{\alpha}(1)}, \ldots, c_{\pi_{\alpha}\left(n_{\alpha}\right)}$ есть $k+2$, то

$$
\left(c_{\pi_{\alpha}(i)}\right)_{i \in N_{n_{\alpha}}}
$$


содержится в $\tau_{\alpha}$ тогда и только тогда, когда он содержится в $\tau_{\alpha}^{\prime}$. Если же

$$
\left(c_{\pi_{\alpha}(i)}\right)_{i \in N_{n_{\alpha}}} \in E_{k+2}^{n_{\alpha}}
$$

To

$$
\left(c_{\pi_{\alpha}(i)}\right)_{i \in N_{n_{\alpha}}}=\left(b_{\pi_{\alpha}(i)}\right)_{i \in N_{n_{\alpha}}} .
$$

Таким образом, с допустим для (2). Поскольку

$$
M_{\tilde{\tau}}(I) \cap N_{n}=M_{\tau^{\prime}}(I)=I,
$$

справедливо равенство $2_{I}(\mathbf{a})=\left.\mathbf{c}\right|_{N_{n}} \in \tau$.

Отношение $\tau$ на $E_{k+3}$ назовем $(k+2)$-центральным, если $\tau$ содержит всякий кортеж, среди компонент которого встречается $k+2$. Отношение $\rho$ назовем прямо неразложимым, если его нельзя представить в виде $\rho=\rho_{1} \times \rho_{2}$.

Для каждого $I \subseteq N_{n}$ такого, что $M_{\tau}(I)=I$, определим $(k+2)$-центральное отношение $\tau(I)$ равенством

$$
\tau(I) \cap E_{k+2}^{|I|}=\left.\tau\right|_{I} \cap E_{k+2}^{|I|}
$$

Пусть также отношение $\tau^{\prime \prime}$ определено формулой

$$
P_{\tau^{\prime \prime}}\left(x_{1}, \ldots, x_{n}\right)=P_{\tau^{\prime}}\left(x_{1}, \ldots, x_{n}\right) \wedge \bigwedge_{I \subseteq N_{n}, M_{\tau}(I)=I} P_{\tau(I)}\left(x_{i}\right)_{i \in I}
$$

где последняя операция берется по $I$, для которых $\left.\tau\right|_{I}$ прямо неразложимо.

Лемма 8. Справедливо равенство $\tau^{\prime \prime}=\tau$.

Доказательство. Докажем включение $\tau^{\prime \prime} \supseteq \tau$. Возьмем $\mathbf{a} \in \tau$. Тогда $\mathbf{a} \in \tau^{\prime}$, поскольку $\tau_{\alpha} \subseteq \tau_{\alpha}^{\prime}$ для всех $\alpha \in \Lambda$. Рассмотрим $I \subseteq N_{n}$ такое, что $M_{\tau}(I)=I$. Если $k+2 \in\left\{a_{i} \mid i \in I\right\}$, то $\left.\mathbf{a}\right|_{I} \in \tau(I)$ в силу $(k+2)$-центральности. В противном случае,

$$
\left.\left.\mathbf{a}\right|_{I} \in \tau\right|_{I} \cap E_{k+2}^{|I|} \subseteq \tau(I) .
$$

Докажем теперь, что $\tau^{\prime \prime} \subseteq \tau$. Возьмем $\mathbf{a} \in \tau^{\prime \prime}$ и пусть

$$
I=\left\{i \mid a_{i} \neq k+2\right\}
$$

По лемме 7 множество $I$ замкнуто, так как $\mathbf{a} \in \tau^{\prime}$. Предположим, что

$$
\left.\tau\right|_{I}=\left.\tau\right|_{I_{1}} \times \ldots \times\left.\tau\right|_{I_{t}}
$$

где все сомножители прямо неразложимы. Поскольку $\left.\mathbf{a}\right|_{I_{j}} \in \tau\left(I_{j}\right)$ для всех $j \in N_{t}$, получаем, что

$$
\left.\left.\mathbf{a}\right|_{I} \in \tau\right|_{I_{1}} \times \ldots \times\left.\tau\right|_{I_{t}}=\left.\tau\right|_{I},
$$

а в силу SN1 справедливо включение $\mathbf{a} \in \tau$. 
Проверим теперь, что для любого $I$ отношение $\tau(I)$ содержится в клоне отношений $\left\langle\mathrm{SN} \cup\left\{\varkappa_{K(\tau, I)}^{r}\right\}\right\rangle$ для любого натурального $r$. Нам потребуется ряд вспомогательных утверждений.

Пусть $\chi-(k+2)$-центральное отношение, не содержащее единичного кортежа, причем множество всех его координат имеет единственную индексацию $\gamma$ ранга $r$. Введем обозначения

$$
n=\operatorname{ar} \chi, \quad \rho=K\left(\chi, N_{n}\right), \quad s=\operatorname{ar} \rho, \quad T=N_{n+s p^{r}} \backslash N_{n} .
$$

Через $\chi^{\downarrow}$ обозначим $\left(n+s p^{r}\right)$-местное отношение, определенное следующими условиями:

(1) для всякого $\mathbf{a} \in \chi^{\downarrow}$ такого, что $\left.\mathbf{a}\right|_{N_{n}} \in E_{k}^{n}$, условие $(i, j) \in \xi\left(\chi, N_{n}\right)$ влечет $a_{i}=a_{j}$, другими словами, $\xi\left(\chi^{\downarrow}, N_{n}\right)=\xi\left(\chi, N_{n}\right)$;

(2) $\mathbf{a} \in \chi^{\downarrow}$ тогда и только тогда, когда

- либо $\left.\mathbf{a}\right|_{N_{n}} \notin E_{k+2}^{n}$ и $\left.\mathbf{a}\right|_{T} \in \varkappa_{v}^{r}$, где $v-s$-местное универсальное отношение,

- либо $\left.\mathbf{a}\right|_{N_{n}}-\gamma$-выделяющий ( $\gamma$-связывающий, $\gamma$-титульный) кортеж с номером $j$ и для всех $i \in N_{n}$ кортеж $\left.\mathbf{a}\right|_{\left\{n+(i-1) p^{r}+1, \ldots, n+i p^{r}\right\}}$ выделяющий (связывающий, титульный) с тем же номером,

- либо $\left.\mathbf{a}\right|_{T}=\mathbf{w}_{\mathbf{b}}^{r},\left.\mathbf{a}\right|_{N_{n}} \in E_{k}^{n}$, причем $K\left(\left.\mathbf{a}\right|_{N_{n}}\right)=\mathbf{b}$,

- либо a $\left.\right|_{N_{n}} \in E_{k+2}^{n} \cap \chi,\left.\mathbf{a}\right|_{N_{n}} \notin E_{k}^{n},\left.\mathbf{a}\right|_{N_{n}}$ - не выделяющий, не связывающий и не титульный кортеж, а $\mathbf{a} \mid T$ содержится в $\varkappa_{v}^{r} \cap E_{k+2}^{|T|}$.

Лемма 9. Справедливо включение $\chi^{\downarrow} \in \mathrm{SN}$.

Доказательство. Обозначим через $\gamma_{0}$ единственную, в силу предложения 2 , индексацию множества $T$. Нетрудно проверить, что оператор $M=M_{\chi \downarrow}$ действует следующим образом: $M(I)=I$, если $N_{n} \nsubseteq I$ и $\gamma_{0}(T \cap I) \neq N_{p^{r}}, M(I)=I \cup T$, если $N_{n} \nsubseteq I$ и $\gamma_{0}(T \cap I)=N_{p^{r}}$, и, наконец, $M(I)=N_{n} \cup T$ в остальных случаях.

Проверим выполнение свойства SN1. Достаточно рассмотреть случай $N_{n} \nsubseteq I$. Возьмем a $\in \chi^{\downarrow}$. Пусть сначала $\gamma_{0}(T \cap I) \neq N_{p^{r}}$. Кортеж $\left.2_{M(I)}(\mathbf{a})\right|_{T}=\left.2_{I}(\mathbf{a})\right|_{T}$ содержится в $\varkappa_{v}^{\rho}$. Поскольку $\left.2_{M(I)}(\mathrm{a})\right|_{N_{n}} \notin E_{k+2}^{n}$, по пункту 2 определения $2_{M(I)}(\mathrm{a}) \in$ $\chi^{\downarrow}$. Если же $\gamma_{0}(T \cap I)=N_{p^{r}}$, то $\left.2_{M(I)}(\mathbf{a})\right|_{T}=\left.\mathbf{a}\right|_{T}$ и в силу пункта 2 определения и $(k+2)$-центральности $\chi$ получаем требуемое.

Проверим SN2. Допустим, что $\left.(k+1, \ldots, k+1) \in \chi^{\downarrow}\right|_{I}$. Поскольку $\left.\chi^{\downarrow}\right|_{N_{n}},\left.\chi^{\downarrow}\right|_{T^{\prime}}$, где $T^{\prime} \subseteq T$ таково, что $\gamma_{0}\left(T^{\prime}\right)=N_{p^{r}}$, не содержат единичного кортежа, множество $I$ не содержит ни $N_{n}$, ни $T$. Но тогда $\left.\chi^{\downarrow}\right|_{I}$ универсально в силу свойства SN1 отношения $\chi^{\downarrow},(k+2)$-центральности $\chi$ и свойства SN2 отношения $\varkappa_{v}^{r}$.

Проверим SN3. Предположим, что $\gamma_{1}, \gamma_{2}$ - индексации $I$ разных рангов. Тогда, в силу того, что $N_{n}$ имеет единственную индексацию, и по предложению $2 N_{n} \nsubseteq I$ и $\gamma_{0}(I \cap T) \neq N_{p^{r}}$. Отсюда, $\left.\chi^{\downarrow}\right|_{I \cap N_{n}}$ и $\left.\chi^{\downarrow}\right|_{I \cap T}$ универсальны согласно пункту 2 определения и свойству SN1 отношения $\varkappa_{v}^{r}$. Кроме того,

$$
\left.\chi^{\downarrow}\right|_{I}=\left.\chi^{\downarrow}\right|_{I \cap N_{n}} \times\left.\chi^{\downarrow}\right|_{I \cap T}
$$

Таким образом, $\left.\chi^{\downarrow}\right|_{I}-$ универсальное отношение. 
Проверим SN4. Пусть $\gamma_{1}, \gamma_{2}$ - индексации множества $I$ и $\gamma_{1}(i) \neq \gamma_{2}(i)$ для некоторого $i \in I$. Тогда $N_{n} \nsubseteq I$, так как в противном случае $I$ однозначно индексируемо. Если к тому же $\gamma_{0}(T \cap I) \neq N_{p^{r}}$, то $\left.\chi^{\downarrow}\right|_{I}$ универсально. В оставшемся случае

$$
\left.\chi^{\downarrow}\right|_{I}=\left.\chi^{\downarrow}\right|_{T \cap I} \times\left.\chi^{\downarrow}\right|_{N_{n} \cap I}=\left.\chi^{\downarrow}\right|_{T \cap I} \times v_{\left|N_{n} \cap I\right|} .
$$

В силу свойств SN3, SN4 отношения $\varkappa_{v}^{r}$ множество $T$ имеет единственную индексацию. Поэтому $i \in N_{n} \cap I$ и требуемое вытекает из указанного равенства.

Проверим SN5. Если $N_{n} \nsubseteq I, \gamma_{0}(T \cap I) \neq N_{p^{r}}$, свойство SN5 тривиально. В случае $N_{n} \nsubseteq I, \gamma_{0}(T \cap I)=N_{p^{r}}$ свойство SN5 следует из SN5 отношения $\varkappa_{v}^{r}$. Пусть $N_{n} \subseteq I$ и $\gamma$ - индексация множества $I$. Положим $\gamma^{\prime}: N_{n} \cup T \rightarrow N_{p^{r}}$, где $\gamma^{\prime}(i)=\gamma(i)$, если $i \in I$, и $\gamma^{\prime}(i)=\gamma_{0}(i)$ в противном случае. Согласно пункту 2 определения ограничение $\gamma^{\prime}$ на $T$ есть индексация $\gamma_{0}$ отношения $\left.\chi^{\downarrow}\right|_{T}$. Поскольку $\chi$ также однозначно индексируемо, по пункту 2 определения получаем требуемое.

Наконец, проверим выполнение свойства SN6. Если $N_{n} \nsubseteq I$, то $K\left(\chi^{\downarrow}, I\right)$ либо универсально (при $\left.\gamma_{0}(I \cap T) \neq N_{p^{r}}\right)$, либо равно $v_{\left|N_{n} \cap I\right|} \times\left.\rho\right|_{J}$, где

$$
J=\left\{j \mid \text { найдется } i \in N_{p^{r}} \text { такой, что } n+(j-1) p^{r}+i \in I\right\}
$$

(при $\gamma_{0}(I \cap T)=N_{p^{r}}$ ). Будем полагать, поэтому, что $N_{n} \subseteq I$. Пусть $S_{1}, \ldots, S_{q}-$ классы эквивалентности $\xi\left(\chi, N_{n}\right)$ и $T_{1}, \ldots, T_{q}$ - классы эквивалентности $\xi\left(\chi^{\downarrow}, T\right)$. Тогда в силу пункта 2 определения классы эквивалентности $\xi\left(\chi^{\downarrow}, I\right)$ суть

$$
S_{1} \cup\left(T_{1} \cap I\right), \ldots, S_{q} \cup\left(T_{q} \cap I\right) .
$$

Наконец, поскольку $\left.\chi^{\downarrow}\right|_{N_{n}}$ и $\left.\chi^{\downarrow}\right|_{T}$ содержат все кодирующие кортежи, получаем, что $K\left(\chi^{\downarrow}, I\right)$ - универсальное отношение.

Лемма 10. Справедливо равенство

$$
P_{\chi}=\exists\left(x_{i}\right)_{i \in T} P_{\chi^{\downarrow}}\left(x_{i}\right)_{i \in N_{n} \cup T} \wedge P_{\varkappa_{\rho}^{r}}\left(x_{i}\right)_{i \in T} .
$$

Для доказательства необходимо лишь заметить, что для любого допустимого кортежа а включение $\left.\mathbf{a}\right|_{N_{n}} \in \chi \cap E_{k}^{n}$ справедливо тогда и только тогда, когда $\left.\mathbf{a}\right|_{T}$ принадлежит $\varkappa_{\rho}^{r} \cap E_{k}^{|T|}$.

Лемма 11. Справедливо включение

$$
\tau(I) \in\left\langle\mathrm{SN} \cup\left\{\varkappa_{K(\tau, I)}^{r}\right\}\right\rangle .
$$

Из лемм 8 и 11 очевидным образом вытекает следующее утверждение.

Предложение 3. Справедливо включение

$$
\begin{aligned}
\tau \in\left\langle\mathrm { SN } \cup \left\{\varkappa_{K(\tau, I)}^{r} \mid I \subseteq N_{\mathrm{ar} \tau}, M_{\tau}(I)=I,\right.\right. \\
\left.\left.\left.\tau\right|_{I} \text { прямо неразложсмо и индексируемо ранга } r\right\}\right\rangle .
\end{aligned}
$$

Далее мы покажем, что если $\left.\tau\right|_{I}$ индексируемо ранга $r$, то

$$
\varkappa_{K(\tau, I)}^{r} \in \in\left\langle\mathrm{SN} \cup\left\{\left.\tau\right|_{I}\right\}\right\rangle \text {. }
$$

Пусть $\gamma$ - индексация (единственная) отношения $\left.\tau\right|_{I}$, и пусть $T_{1}, \ldots, T_{q}-$ классы эквивалентности $\xi\left(\left.\tau\right|_{I}\right)$. Пусть также $s=\operatorname{ar} K(\tau, I)$. Положим $\pi: I \rightarrow N_{s p^{r}}$, причем $\pi(i)=(u-1) p^{r}+\gamma(i)$, если $i \in T_{u}$. 
Лемма 12. Пусть $\tau_{1}=\varkappa_{v}^{r}$, где $v$ - универсальное s-местное отношение, $\tau_{2}=\varkappa_{K(\tau, I)}^{r}$. Toгda

$$
P_{\tau_{2}}\left(x_{1}, \ldots, x_{s p^{r}}\right)=P_{\tau_{1}}\left(x_{1}, \ldots, x_{s p^{r}}\right) \wedge P_{\tau \mid I}\left(x_{\pi(i)}\right)_{i \in I}
$$

Доказательство. Пусть $Q$ - образ отображения $\pi$, пусть также $\gamma^{\prime}: Q \rightarrow N_{p^{r}}$ такое, что $\gamma^{\prime}(j)=\gamma(i)$, если $\pi(i)=j$. По построению $\pi$ отображение $\gamma^{\prime}$ определено корректно и, более того, $\gamma^{\prime}(j) \equiv j\left(\bmod p^{r}\right)$. По предположению $\left.(k+1, \ldots k+1) \notin \tau\right|_{I}$ и $\left.\tau\right|_{I}$ индексируемо, поэтому $\gamma^{\prime}(Q)=N_{p^{r}}$. Пусть еще отображение $\gamma_{0}: N_{s p^{r}} \rightarrow N_{p^{r}}$ таково, что $\gamma_{0}(i) \equiv i\left(\bmod p^{r}\right)$. Очевидно, $\gamma^{\prime}=\left.\gamma_{0}\right|_{Q}$.

Пусть сначала $\mathbf{a} \in \tau_{2}=\varkappa_{K(\tau, I)}^{r}$. Тогда очевидно, что $\mathbf{a} \in \varkappa_{v}^{r}$. Положим

$$
J=\left\{i \mid a_{i} \neq k+2\right\} .
$$

Если $\gamma_{0}(J) \neq N_{p^{r}}$, то $Q \nsubseteq J$ и $\left.\left(a_{\pi(i)}\right)_{i \in I} \in \tau\right|_{I}$ в силу свойства SN1 отношения $\left.\tau\right|_{I}$. Если а - выделяющий, связывающий или титульный кортеж, то таков и кортеж $\left.\mathbf{a}\right|_{Q}$ относительно индексации $\gamma^{\prime}$. Отсюда, $\left.\left(a_{\pi(i)}\right)_{i \in I} \in \tau\right|_{I}$. Наконец, если а - кодирующий кортеж, то

$$
K(\mathbf{a}) \in K(\tau, I)=K\left(\left.\tau\right|_{I}, I\right),
$$

поэтому кортеж $\left(a_{\pi(i)}\right)_{i \in I}$ содержится в $\left.\tau\right|_{I}$.

Обратно, предположим, что $\mathbf{a} \in \varkappa_{v}^{r}$ и $\left.\left(a_{\pi(i)}\right)_{i \in I} \in \tau\right|_{I}$. Поскольку a $\in \varkappa_{v}^{r}$, достаточно рассмотреть случай, когда а - кодирующий кортеж. Как и выше, из того, что $\left.\left(a_{\pi(i)}\right)_{i \in I} \in \tau\right|_{I}$ следует включение

$$
K(\mathbf{a}) \in K\left(\left.\tau\right|_{I}, I\right)=K(\tau, I) .
$$

Отсюда немедленно получаем, что $\mathbf{a} \in \tau_{2}$.

Таким образом, если $I$ замкнуто, прямо неразложимо и индексируемо ранга $r$, то $\varkappa_{K(\tau, I)}^{r} \in\left\langle\mathrm{SN} \cup\left\{\left.\tau\right|_{I}\right\}\right\rangle$.

Следующее предложение непосредственно вытекает из взаимной однозначности $\psi$, предложения 3 и только что доказанного.

Предложение 4. Для любих последовательностей $\mathrm{C}_{1}, \mathbf{C}_{2}$

$$
\psi\left(\mathbf{C}_{1} \wedge \mathbf{C}_{2}\right)=\psi\left(\mathbf{C}_{1}\right) \wedge \psi\left(\mathbf{C}_{2}\right)
$$

Доказателъство. Включение $\psi\left(\mathbf{C}_{1} \wedge \mathbf{C}_{2}\right) \subseteq \psi\left(\mathbf{C}_{1}\right) \wedge \psi\left(\mathbf{C}_{2}\right)$ очевидно. Если $\tau \in$ $\psi\left(\mathbf{C}_{1}\right) \wedge \psi\left(\mathbf{C}_{2}\right)$, то $K(\tau, I) \in \mathbf{C}_{1} \wedge \mathbf{C}_{2}$ для всякого индексируемого множества $I \subseteq N_{\operatorname{ar}}$. Отсюда, по предложению 3 и лемме $12 \tau \in \psi\left(\mathbf{C}_{1} \wedge \mathbf{C}_{2}\right)$.

Проверим, наконец, что образом отображения $\psi$ является интервал в решетке $\mathscr{L}_{k+2}^{\partial}$.

Предложение 5. Если $\mathbf{C}^{1} \leqslant \mathbf{C}^{2} u \psi\left(\mathbf{C}^{1}\right) \leqslant C \leqslant \psi\left(\mathbf{C}^{2}\right)$, по $C=\psi\left(\mathbf{C}^{\prime}\right)$ для некоторой последовательности $\mathbf{C}^{\prime}$.

Дохазательство. Пусть $T_{r}-$ множество всех таких $\rho$, что $\rho=K(\tau, I)$, отображение $\tau \in C \backslash \psi\left(\mathbf{C}^{1}\right)$, где множество $I$ индексируемо ранга $r$ и

$$
T=\bigcup_{r \in \mathbf{N}} T_{r}
$$


Для любого $\rho \in T_{r}$ по лемме 12 справедливо включение $\varkappa_{\rho}^{r} \in C$. Положим $\mathbf{C}^{\prime}=\left(C_{1}^{\prime}, C_{2}^{\prime}, \ldots\right)$, где $C_{r}^{\prime}$ порожден множеством $C_{r}^{1} \cup T_{r}, r=1,2, \ldots$ Тогда

$$
\psi\left(\mathbf{C}^{\prime}\right) \subseteq\left\langle\psi\left(\mathbf{C}^{1}\right) \cup\left\{\varkappa_{\rho}^{r} \mid r \in \mathbf{N}, \rho \in T_{r}\right\}\right\rangle .
$$

С другой стороны, каждое $\tau \in C$ представимо формулой, в которой участвуют лишь отношения

$$
\tau_{\alpha} \in \mathrm{SN} \cup\left\{\varkappa_{\rho}^{r} \mid \rho \in C_{r}^{2}, r \in \mathrm{N}\right\}, \quad \alpha \in \Lambda .
$$

В силу предложения 3 можно считать, что все кодирующие отношения $\rho_{\alpha}$ содержатся в $\left\{\varkappa_{\rho}^{r} \mid \rho \in C_{r}^{\prime}, r \in \mathbf{N}\right\}$. Итак, $\rho \in \psi\left(\mathbf{C}^{\prime}\right)$.

Это завершает доказательство теоремы 1.

\section{Список литературы}

1. Post E., Two-valued Iterative Systems of Mathematical Logic. Ann. Math. Stud. 5, Princeton Univ. Press, Princeton, 1941.

2. Булатов А. А., Конечные подрешетки в решетке клонов. Алгебра и логика (1994) 33, №5, 514-549.

3. Булатов А. А., Тождества в решетках замкнутых классов. Дискретная математика (1992) 4, №4, 140-148.

4. Булатов А. А., Подрешетки решетки клонов функций на трехэлементном множестве. I. Алгебра и логика (1999) 38, №1, 3-23.

5. Гретцер Г. Общал теория решеток. Мир, Москва, 1982.

6. Яблонский С. В., Функциональные построения в $k$-значной логике. Труды Матем. инта им. В. А. Стехлова (1958) 51, 5-142.

7. Pöschel R., Kalužnin L. A., Funktionen- und Relationenalgebren. Ein Kapitel der diskreten Mathematik VEB DVW, Berlin, 1979.

8. Янов Ю. И., Мучник А. А., О существовании $k$-значных замкнутых классов, не имеющих конечного базиса. Докл. АН СCCP (1959) 127, №1, 44-46.

9. Haddad L., Rosenberg I. G., Un intervalle Booléen de clones sur un univers fini. Ann. Sci. Math. Québec (1988) 12, №2, 211-231.

10. Крохин А. А., Моноидальные интервалы в решетках клонов. Алгебра и логика (1995) 34, №3, 288-310.

11. Боднарчук В. Г., Калужнин Л. А., Котов В. Н., Ромов Б. А., Теория Галуа для алгебр Поста. I, II. Кибернетика (1969) 3, 1-10; 5, 1-9.

Статья поступила 25.05.1998. 